\title{
Centennials en TikTok: tipología de vídeos. Análisis y comparativa España- Gran Bretaña por género, edad y nacionalidad
}

\section{Centennials on TikTok: type of video. Analysis and comparative Spain- Great Britain by gender, age and nationality}

Rebeca Suárez-Álvarez. Universidad Rey Juan Carlos. España.

rebeca.suarez@urjc.es

$[\mathrm{CV}]$ (1) $\mathrm{R}^{\circ}$

Antonio García-Jiménez. Universidad Rey Juan Carlos. España.

antonio.garcia@urjc.es

$[\mathrm{CV}] \odot \mathrm{R}^{\mathrm{s}}$

Este artículo se circunscribe en el proyecto "Nuevos escenarios de vulnerabilidad digital: alfabetización mediática para una sociedad inclusiva" PROVULDIG-2-CM (ref. H2019/HUM5775), financiado por la CAM y el Fondo Social Europeo (01/01/2020-31/12/2022). El proyecto está vinculado GICOMSOC, grupo de investigación de la Universidad Rey Juan Carlos.

Cómo citar este artículo / Referencia normalizada

Suárez-Álvarez, R., García-Jiménez, A. (2021). Centennials en TikTok: tipología de vídeos. Análisis y comparativa España-Gran Bretaña por género, edad y nacionalidad. Revista Latina de Comunicación Social, 79, 1-22. https://www.doi.org/10.4185/RLCS-2021-1503

\section{RESUMEN}

Introducción: TikTok es una red social nativa digital que ha logrado generar el interés entre los adolescentes pero ¿qué tipo de contenidos crean y difunden en esta aplicación? Abordamos esta pregunta con el objetivo de conocer la tipología de vídeos que están subiendo los tiktokers adolescentes españoles y británicos a sus perfiles en esta red social. Metodología: Mediante metodología de análisis de contenido se estudian 447 vídeos de 12 tiktokers británicos y 12 españoles chicos y chicas de entre 11 y 17 años con más de medio millón de seguidores, con una visión individual y comparada de ambas nacionalidades. Resultados: Los contenidos que crean y difunden ambos géneros y nacionalidades son videoselfies, coreografías y vídeos con finalidad divertida. Los resultados muestran que los adolescentes de 16 y 17 años son los más activos en esta red social. Españoles y británicos crean y difunden vídeos con contenidos similares, siendo la edad, y no el género, la variable determinante para su producción digital. Se observa que los chicos abandonan los videojuegos para dar lugar a una auto-representación más protagónica. Discusión y conclusiones: Se observa una carencia en la pluralidad del tipo de contenidos que crean y difunden en TikTok ambos géneros y nacionalidades. Resulta interesante la atenuación de los tradicionales roles de género que los centennials muestran en sus producciones audiovisuales en TikTok, lo que refleja la posible evolución de su comportamiento de género en las redes sociales.

PALABRAS CLAVE: TikTok; redes sociales; centennials, adolescentes; género; edad; consumo 


\begin{abstract}
Introduction: TikTok is a digital native social network has managed to generate interest among teenagers through which they share their audiovisual productions from mobile devices, but what type of content do teenagers create and disseminate on this application? We address this issue with the aim of finding out the type of videos that Spanish and British teenage tiktokers are uploading to this social network. Methodology: Using content analysis methodology, 447 videos from 12 British and 12 Spanish tiktokers aged between 11 and 17 with more than half a million followers are studied. It provides an analysis by nationality and a comparative view of both nationalities. Results: Contents created and disseminated by both genders and nationalities are videoselfies, choreographies and videos with a fun purpose. Results show 16 and 17-year-old are the most active on this social network. Spanish and British teenagers create and disseminate videos with similar content. They show is age, not gender, determining variable for their digital production. It is observed boys abandon videogames to give way to a more protagonist self-representation in this social network. Discussion and conclusions: There is a lack of plurality in the type of content created and disseminated on TikTok by both genders and nationalities. It is interesting to note the attenuation of the traditional gender roles adolescents show in their audio-visual productions on TikTok, which reflect the possible gender-related development into their behavior on social networks.
\end{abstract}

KEYWORDS: TikTok; social networks; centennials; teenagers; gender; age; consume

\title{
CONTENIDO
}

1. Introducción. 1.1. TikTok y adolescentes. 1.2. Diferencias por edad, género y nacionalidad. 2. Objetivos y preguntas de investigación. 3. Metodología. 3.1. Criterios de selección de la muestra; 3.2. Codificación de variables. 4. Resultados. 4.1. Tipología de contenidos de tiktokers españoles. 4.2. Tipología de contenidos de tiktokers británicos. 4.3. Comparativa tiktokers españoles-británicos: género, edad y nacionalidad en los vídeos creados y difundidos. 5. Conclusiones y discusión. 6. Futuras investigaciones. 7. Bibliografía 8. Currículum Vitae

\section{Introducción}

Las redes sociales de vídeos se han convertido en los nuevos canales de comunicación de los centennials, adolescentes, que más allá de la delimitación etaria que efectúan McGorry y McGorry, (2017) y Sharma (2019) que tratan de circunscribirlos entre 1997 hasta aproximadamente 2015, son una generación nativa digital para la que las tecnologías digitales son parte natural de su mundo, a las que acceden desde distintos dispositivos a diario y por las que se sienten influenciados (Qasem, 2021; Smith \& Mills, 2019). En ellas comparten sus vidas a través de sus producciones audiovisuales en una creciente hiperconectividad (Zlamal, et al., 2020). Algunas de estas plataformas han perecido como Vine (reconvertida en Byte), y Lasso, una suerte de copia de TikTok que cerró en julio de 2020 (López, 2020; Martín, 2020). Otras como YouTube, Instagram o Facebook han logrado mayor éxito.

La última en emerger, en 2018, es la aplicación de social media TikTok que ha logrado más de 800 millones de usuarios activos mensuales (We Are Social y Hootsuite, 2020) de los cuales el 90\% acceden a la aplicación diariamente (Sensor Tower, 2020). TikTok se ha convertido en la séptima red social a nivel mundial y la única que no pertenece al grupo empresarial de Facebook. En 2020 ha registrado un incremento del 60\% de usuarios únicos (We Are Social y Hootsuite, 2019) y durante el confinamiento total en España y parcial en Gran Bretaña (UK), en marzo de 2020, la plataforma fue la aplicación no relacionada con videojuegos más descargada a nivel mundial con más de 115,2 
millones de descargas, lo que representa un aumento del 98,4\% con respecto al año anterior (Sensor Tower, 2020).

En España y UK, TikTok fue la aplicación de redes sociales más descargada durante 2020 (Sensor Tower, 2020). En UK quedó por delante de Instagram y Snapchat (We are social, 2020) y en España adelantó a WhatsApp y a Instagram (Statista, 2020a). Durante marzo-abril de 2020, la plataforma incrementó su consumo un $150 \%$ con respecto al año anterior hasta alcanzar los 71 minutos en 2020, convirtiéndose en competencia directa con YouTube en la que los menores pasaron 75 minutos al día (Qustoio, 2020). En Reino Unido, el 13\% de los menores de entre 12 y 15 años utilizan TikTok (The Office of Communications [Ofcom], 2020) con un tiempo medio diario de 41 minutos frente a los 75 de YouTube (Statista, 2020b). Cifras que, al igual que en España, se han incrementado durante la crisis sanitaria de la COVID-19 superando a YouTube con una media de visionado diario de 83 minutos entre los adolescentes de este país (Qustodio, 2020). Esta predilección de los adolescentes por esta red social puede derivar en una potencial migración de los adolescentes de una red social a otra (Marcelino, 2015) o en la más previsible situación actual centrada en la posesión de perfiles en varias redes sociales dando lugar a la sobreexposición (Gonzales y Hancock, 2011).

El interés de los adolescentes por esta red social de vídeos cortos, fortalecido por el contexto digital en el que están creciendo (Bleakley, Ellithorpe y Romer, 2016), conduce a investigar qué contenidos están compartiendo en esta red social, como así ha ocurrido desde distintas aproximaciones con YouTube (García y Montes, 2020), Instagram (Anggreni, 2019), Facebook (Botou y Marsellos, 2018), Snapchat (Bruna, 2020) o estudios combinados con Vine y YouTube (Yarohs, et al, 2016).

\subsection{TikTok y adolescentes}

En TikTok los usuarios conocidos como tiktokers van cargando sus perfiles de contenidos creados por ellos mismos como productores culturales (Hubermann, 2020) en la que los adolescentes expresan sus opiniones y construyen su identidad de forma performativa (Shutsko, 2020). Estudiando estas prácticas como productores audiovisuales, la Teoría de la Acción Social de Goffman (2009) sirve como aproximación teórica de la presente investigación para abordar las temáticas que crean y difunden los adolescentes en las redes sociales, y que Yarohs, et al., (2016) en su análisis de contenido en Vine y YouTube, McRoberts et al., (2017) en Snapchat y Djafarova y Trofimenk (2017) en Instagram abordan en el comportamiento y puesta en escena del yo performativo de los adolescentes.

La auto-producción de los adolescentes en TikTok se basa en la interpretación de vídeos cortos basados en fragmentos musicales (Klug, 2020) que ha eclosionado en una especie de zona de juegos visual (Anderson, 2020) en la que los adolescentes estimulan la prácticas de remezclar música (Bresnick, 2019) y de aprender habilidades como interpretar y realizar movimientos de baile (Ahlse, Nilsson y Sandström, 2020). Bossen y Kottasz (2020), Wang (2020a) y Yang, Zhao y Ma (2019) señalan el entretenimiento, la socialización y la auto-expresión creativa como los principales predictores de su consumo y producción de contenidos en TikTok. En ellas, los adolescentes mitigan su necesidad de autoexpresión compartiendo contenidos, aspecto acerca del que Van Dijck, (2019) se muestra crítico puesto que considera que los adolescentes usan las redes sociales principalmente como herramientas de autopromoción personal en las que muestran sus estilos de vida y personalidad con el objetivo de ofrecer a sus seguidores contenidos que les interesen (Pedraza y Villagarra, 2019). López de Ayala y Paniagua (2019) les otorgan finalidades colaborativas basadas en la necesidad de influir en los demás y de sentirse útiles para la sociedad. 
Bossen y Kottasz (2020) estudian el uso de TikTok por pre-adolescentes y adolescentes y señalan que los pre-adolescentes son usuarios más activos que los adolescentes en esta red social en la que buscan además el reconocimiento social y la búsqueda de fama como elementos de carácter persuasivo para su uso. Patel y Binjola (2020) también concluyen que esta plataforma facilita la expresión creativa y el fomento del talento al permitir que los adolescentes se muestren como celebrities. En esta misma línea de trabajo, Leight (2019) señala que TikTok les resulta atractivo por la oportunidad que les ofrece de convertirse en famosos rápidamente. Nuevas celebridades digitales con las que se sienten más identificados que con los famosos tradicionales como refrendan Djafarova y Rushworth (2019) en su estudio de los perfiles "Instafamous" en Instagram. No obstante, Bossen y Kottasz (2020) señalan que en TikTok predominan los comportamientos de consumo pasivo, condiciendo con las apreciaciones de Edelmann (2017) que apunta el hecho de que la mayor parte de los internautas de redes sociales no produce contenidos, sino que observa y visualiza los que ofrecen otros, comportamientos igualmente valiosos que les convierten en "merodeadores" de la actividad digital (Gazit, et al., 2018; Romero-Hall, 2017).

Resulta escasa la literatura centrada en analizar de forma cuantitativa y desde una perspectiva comparada de dos nacionalidades la tipología de vídeos que están compartiendo los adolescentes en TikTok, lo que pone de manifiesto la necesidad de esta investigación. Sí hay estudios vinculados a los contenidos que están compartiendo los adolescentes en sus perfiles de otras redes sociales. TurViñes, Núñez-Gómez y González-Río (2021) en su investigación en los 5 canales españoles de menores con mayor número de visitantes y suscriptores de YouTube señalan que la mayoría de los vídeos que publican se centran en juegos, narraciones, mostrar productos, desafíos y tutoriales. Yarohs, et al., (2016) han trabajado con la identificación de los contenidos que comparten los adolescentes como creadores de contenidos en las redes sociales YouTube y Vine. En YouTube destacan los vídeos en los que realizan actividades cotidianas (44\%), videoselfies (25\%) y tutoriales (14\%); mientras que en Vine se decantan por compartir contenidos centrados en coreografías (62\%), vídeos divertidos solos o acompañados $(41 \%)$ y contenidos creados a partir de diversas fuentes (11\%). En Instagram, Sulistyo (2018) señala que los adolescentes crean vídeos elaborados con finalidad de entretener cuya temática se centra en vídeos informativos, educativos y de promoción de productos o servicios. Además, señala que los adolescentes toman como inspiración para sus producciones audiovisuales instagramers que consideran exitosos, en línea con las conclusiones de Sherman, et al., 2108 y Sherman, et al., 2016 que en la misma red social confirman la influencia de los pares en la creación y difusión de contenidos de los adolescentes con conexiones virtuales compartidas.

Una cuestión relevante, aunque no entra dentro de los objetivos directos de este trabajo, es la referida a las narrativas que se incorporan en los vídeos de las rede sociales. Como sugiere González (2018), en algunas de ellas, la narrativa audiovisual digital se fusiona con la estética caracterizándose por la discontinuidad, la interactividad, el dinamismo, la vitalidad, la creación de mundos etéreos y efímeros y el fomento de comunidades virtuales (Holtzman, 1997). Cualidades que continúan vigentes y a las que Suing, Carpio y Sinche (2020) añaden la tolerancia a la baja calidad de los contenidos, Pérez-Rufí (2018) la innovación del lenguaje para utilizarlo de forma creativa y Ródenas, Ferreras y Torrado (2021) la ruptura con la unidireccionalidad y su elaboración mediante diversos dispositivos y tecnologías. Además, estas unidades creativas aportan significados de la realidad en función de la interacción y la participación que se genera a raíz de ellas (Jauregui \& Ortega, 2020) convirtiendo estos relatos en nuevos mundos y experiencias en las que todos pueden participar, escuchar y ser escuchados (Costa, 2013).

\subsection{Diferencias por edad, género y nacionalidad}


Montes-Vozmediano, García-Jiménez y Menor-Sendra (2018) certifican la relación entre la autoría de los vídeos y el tipo de contenido que consumen los adolescentes y señalan que los vídeos de los youtubers adolescentes se centran principalmente en contenidos divertidos, coreografías, remezclas y selfies. Apuntan que los vídeos de los adolescentes youtuber son los más vistos, los que mayor impacto obtienen y cuya finalidad es eminentemente divertida. Haddon y Vicent (2015) señalan que el tipo de consumo se modifica y evoluciona con la edad de los menores y García-Jiménez, Lópezde-Ayala López y Montes-Vozmediano (2020) confirman que la edad se muestra como una variable de referencia en el acceso a las redes sociales como WhatsApp, Instagram y YouTube, coincidiendo en su socialización y entretenimiento como principales motivaciones de uso.

La producción, consumo y difusión de contenidos de ocio en redes sociales registran diferencias en relación al género de los adolescentes (Fernández-de-Arroyabe, Lazkano-Arrillaga, y EguskizaSesumaga, 2018). Bossen y Kottasz (2020) han observado que las niñas pre-adolescentes son las mayores usuarias de TikTok con comportamientos pasivos de consumo (visionado), participación (me gusta y compartir contenidos) y contribución de contenidos. Desde el punto de vista femenino, Wang (2020b) investiga las estrategias de Personal Branding de las chicas en TikTok para lograr atraer a seguidores y destaca que las chicas preferencialmente comparten vídeos con fondos simples con ropa casual, aunque detecta diferencias entre las chicas chinas que se decantan por usar complicadas técnicas de edición en sus vídeos en Douyin (TikTok en China) y las americanas que prefieren ediciones más simples. En la misma red social, Khattab (2019) estudia las expresiones faciales, corporales y la indumentaria de los usuarios de tres desafíos de TikTok y concluye que TikTok ilustra la normalización de las imágenes corporales estereotipadas de belleza y género.

En otras redes sociales, Booker, Kelly y Sacker (2018) señalan diferencias de género en el uso de las redes sociales y el bienestar en adolescentes de entre 10 y 15 años en UK y ponen de relieve diferencias importantes entre chicos y chicas. Afirman que el uso intensivo de las redes sociales en la adolescencia temprana tiene implicaciones en su desarrollo socioemocional en la adolescencia tardía, especialmente en las chicas. En los chicos no aprecian dicha relación y aluden a su preferencia por los videojuegos frente a la mayor interacción en las redes sociales de las chicas como también señalan Witt, Massman y Jackson (2011) en su investigación sobre las tendencias en el uso de videojuegos por parte de los adolescentes.

Maloney, Roberts y Caruso (2017) y Blanco-Ruiz y Sainz-de-Baranda (2018) concluyen que en YouTube son los chicos los que lideran las facetas creativas y de producción de vídeo, y Regueira, Alonso-Ferreiro y Da-Vila (2020) confirman que son los canales de los chicos los que mayor número de suscriptores y visualizaciones logran con una menor representación femenina en las cuentas de Instagram de los 50 youtubers con más éxito de España. Coinciden con Wotanis y MacMillan (2014) en la presencia de desigualdades de género en esta red social, la perpetuación de los roles de estereotipo de género y de una mayor interacción social en el caso de las chicas que centran su producción en fotografías y vídeos de ellas mismas frente a ellos que se decantan por la producción y visualización de videojuegos.

En relación con la nacionalidad, Catalina-García, Sousa y Sousa (2019) en su estudio con estudiantes de Brasil, España y Portugal sobre su comportamiento político-social en redes sociales señalan que, aunque la nacionalidad no segmenta a los jóvenes por redes sociales, detectan diferencias en cuanto a la preferencia por una u otra red social: los portugueses se decantan por poseer perfiles en Instagram y en YouTube, los brasileños en Facebook y los españoles en Twitter, siendo YouTube la red social a la que le otorgan mayor credibilidad, especialmente los portugueses. Luque, Tejedor y Bugs (2017) señalan que las principales motivaciones para el uso de las redes sociales por los españoles y los latinoamericanos es la comunicación con la que conforman un vínculo de pertenencia a una identidad 
colectiva, no ser excluidos del grupo y lograr el reconocimiento de los demás en el contexto virtual. No se han encontrado estudios que analicen los contenidos que crean y difunden los adolescentes españoles y británicos atendiendo a sus diferencias por nacionalidad.

\section{Objetivos y preguntas de investigación}

El objetivo de la presente investigación es determinar los tipos de contenidos que los adolescentes publican en TikTok ofreciendo una dimensión comparada entre los adolescentes de España y UK. Ambos países han sido seleccionados por sus diferencias culturales, económicas y socio-políticas para estudiar si los centennials españoles y británicos comparten contenidos similares o si dichas diferencias determinan los contenidos que crean y difunden en esta red social. Se investiga determinar cuáles son las temáticas que más les interesan teniendo en cuenta su género, nacionalidad y edad, puesto que los pre-adolescentes pertenecientes a la adolescencia temprana (10-13 años), mediana (14-16 años) y tardía (17-19 años) (Fondo de las Naciones Unidas para la Infancia [UNICEF], 2020) no se comportan de igual forma.

Los productores de los vídeos son adolescentes que suben y difunden sus contenidos audiovisuales en un afán de ganar mayor cuota de protagonismo (Aguaded y Sánchez, 2013) en unas creaciones propias, ágiles y cortas. Se está investigando sobre qué contenidos están compartiendo los adolescentes en otras redes sociales, pero todavía no se ha profundizado en la red social TikTok teniendo en cuenta la género, edad y nacionalidad de los autores. Resulta necesario estudiar qué tipología de vídeos están subiendo entendidas como aquellas temáticas a las que los adolescentes les resultan interesantes como vía de comunicación con sus iguales (Lenhart, 2015). Para ello, se plantean las siguientes preguntas:

- P1. ¿Qué tipo de contenidos crean y suben los tiktokers españoles adolescentes a TikTok y existen diferencias significativas en el tipo de contenidos según su edad y género?

- P2. ¿Qué tipo de contenidos crean y suben los tiktokers británicos adolescentes a TikTok y existen diferencias significativas en el tipo de contenidos según su edad y género?

- P3. ¿Las variables de género, edad y nacionalidad influyen en el tipo de contenidos que suben a TikTok los adolescentes?

\section{Metodología}

Para la investigación se ha aplicado metodología de análisis de contenido (Baños-González, Canorea y Rajas, 2020; Krippendorff, 2018) como método científico cuantitativo que permite evaluar los mensajes digitales (McMillan, 2009; Richards, 2020) difundidos por adolescentes españoles y británicos para establecer y conocer qué tipos de contenidos audiovisuales aportan a esta red social. La investigación realizada es de carácter exploratorio-descriptivo para identificar prácticas y estrategias de los usuarios de los vídeos que los adolescentes crean. Su escenificación cotidiana en la creación de vídeos y participación activa en la red social permite conocer cómo se auto-representan en según la tipología de contenidos audiovisuales que deciden compartir en sus canales de TikTok.

Se emplean las pruebas estadísticas de análisis de la varianza ANOVA unifactorial para evaluar la relevancia de uno o más factores para comparar las medias de las variables de respuesta, ampliada con la versión multivariada con el test Brown-Forsythe. Asimismo, se aplica el coeficiente de correlación de Pearson con la prueba Chi Cuadrado que permite contrastar y confirmar las frecuencias observadas con las esperadas con el objetivo de garantizar la validez y fiabilidad de los resultados.

\subsection{Criterios de selección de la muestra}


La unidad muestral del estudio son los vídeos que han subido los adolescentes a esta red social. La selección de la muestra comprende 447 vídeos de 12 cuentas de adolescentes españoles y 12 de adolescentes de UK de entre 11 y 17 años desde el 6 de enero al 29 de octubre de 2020. La horquilla temporal de la muestra se formaliza siguiendo las publicaciones de los adolescentes hasta llegar a los 20 vídeos en cada cuenta, excepto en el caso de una chica británica que aporta 17 vídeos a esta red social durante el periodo de tiempo estudiado.

Para realizar el estudio comparado por género y nacionalidad se seleccionaron cuentas pertenecientes a 6 chicos y 6 chicas de ambas nacionalidades siguiendo el criterio de número de seguidores (más de medio millón) y que sean conocidos por su actividad en esta red social, y no por otras profesiones populares como cantantes o actores. Con el objetivo de estudiar vídeos que hayan generado interés en la audiencia, cada unidad muestral alcanza más de mil reproducciones cada una. Cifra que además resulta determinante puesto que a partir de ella, los adolescentes pueden comenzar a monetizar sus producciones audiovisuales en redes sociales según la métrica de ingresos que calcula la tasa RPM (Revenue Per Thousand).

El género de los tiktokers se ha comprobado mediante la visualización de todos los vídeos. La edad y la nacionalidad se han confirmado a través de los buscadores Google y Bing, del registro de la información que los adolescentes incluyen en sus cuentas y en la página web https://es.famousbirthdays.com que recoge información actualizada de celebridades de TikTok.

Tabla 1. Selección de la muestra

\begin{tabular}{|l|c|c|c|c|}
\hline \multicolumn{1}{|c|}{ Cuenta del usuario } & Edad & Género & Nacionalidad & $\mathrm{N}^{\mathrm{o}}$ seguidores \\
\hline @ kylethomas & 16 & chico & inglesa & $17,7 \mathrm{M}$ \\
\hline @ markanastasio & 16 & chico & inglesa & $8,6 \mathrm{M}$ \\
\hline @Its.bellido & 16 & chica & española & $6 \mathrm{M}$ \\
\hline @ maxandharveyveryofficial & 16 & chico & inglesa & $6 \mathrm{M}$ \\
\hline @ariannmusic & 14 & chica & española & $6,4 \mathrm{M}$ \\
\hline @ annemoda14 & 14 & chica & española & $3,7 \mathrm{M}$ \\
\hline @martindant & 15 & chica & española & $3,6 \mathrm{M}$ \\
\hline @ charloteelooks & 17 & chica & inglesa & $3.5 \mathrm{M}$ \\
\hline @ havannawinter & 14 & chica & inglesa & $3,3 \mathrm{M}$ \\
\hline @ sietexx & 17 & chico & española & $2,9 \mathrm{M}$ \\
\hline @ hanamartinx & 17 & chica & inglesa & $2,6 \mathrm{M}$ \\
\hline @aynoficial & $15 \mathrm{y} 17$ & chico & española & $2,3 \mathrm{M}$ \\
\hline @abe.oficial & 17 & chico & española & $2 \mathrm{M}$ \\
\hline @ cesar_abril & 17 & chico & española & $1,8 \mathrm{M}$ \\
\hline @emandloz & 17 & chica & inglesa & $1,8 \mathrm{M}$ \\
\hline @valeriaaliti & 17 & chica & española & $1,6 \mathrm{M}$ \\
\hline @jooaquinrs & 16 & chico & española & $1,5 \mathrm{M}$ \\
\hline @joosejulioo_002 & 14 & chico & española & $1,5 \mathrm{M}$ \\
\hline @ danwolley & 17 & chico & inglesa & $1,3 \mathrm{M}$ \\
\hline @ connxr_r & 11 & chico & inglesa & $985,8 \mathrm{~K}$ \\
\hline @ohyeahelijah & chico & inglesa & $860,4 \mathrm{~K}$ \\
\hline @tianawilson & chica & inglesa & $765,4 \mathrm{~K}$ \\
\hline @babypecas & 17 & española & $685,6 \mathrm{~K}$ \\
\hline
\end{tabular}


Fuente: elaboración propia

La cuenta @aynoficial pertenece a dos hermanos varones españoles que comparten canal y en todos los vídeos aparecen los dos. Estos hermanos tienen edades diferentes (el pequeño tiene 15 años y el mayor 17 años). Para garantizar un análisis estadístico riguroso en el que ambas edades figuren en el estudio, se ha duplicado el número de vídeos en ambos grupos de edad: 20 vídeos para el grupo de 15 años y 20 vídeos para el grupo de edad de 17 años. Durante el análisis de la muestra no se ha encontrado ninguna chica de 13 años de ambas nacionalidades que cumpla con los criterios de selección por lo que quedan excluidas del estudio (Tabla 1).

\subsection{Codificación de variables}

Basadas en Yarosh, et al., (2016) y García y Montes (2020) se ha realizado y adaptado la categorización de las variables registradas para las que se diseñó una cédula de análisis estructurada en dos secciones. La primera registra el nombre del canal, la fecha de publicación, título, género del autor y su edad, y la segunda la tipología de contenidos.

La tipología de los vídeos o tipo de contenidos se segmenta en ocho categorías atendiendo a las investigaciones estudiadas:

1. Videojuegos en los que los adolescentes juegan, comentan y muestran sus destrezas y habilidades en el desarrollo de objetivos y logros trazados por los videojuegos. Por ejemplo, el vídeo del tiktoker @ cesar_abril (https://bit.ly/3ut7ApI), teniendo en cuenta que comparte otras categorías de análisis.

2. Coreografías o vídeos musicales en los que los y las protagonistas bailan e interpretan las canciones empoderándose como intérpretes del contenido que se escucha y visualiza, y que ellos mismos han seleccionado. Teniendo en cuenta que TikTok es una red social cuya base es la música, se decidió otorgarle una categoría propia con el fin de averiguar si los adolescentes están utilizando esta red social para algo más que para bailar y cantar, como se demuestra en los resultados obtenidos. Por ejemplo, el vídeo de @ havannawinter (https://bit.ly/3fYCn9w), teniendo en cuenta que comparte otras categorías de análisis.

3. Vídeos que recogen escenas convencionales que se desarrollan sin un guion o planificación previa en la que el adolescente realiza algún actividad cotidiana de su día a día como puede ser estudiar, ducharse o lavarse los dientes. Por ejemplo, el vídeo de @jooaquinrs (https://bit.ly/3dEv8Rw), teniendo en cuenta que comparte otras categorías de análisis.

4. Vídeos divertidos en los que se recogen la reproducción de actividades atípicas cuyo propósito es entretener y divertir a la audiencia a través de acciones orientadas a tal fin basadas en actitudes activas, divertidas y desenfadadas así como bromas y mofas cuyo propósito es evocar el contento en el público. Por ejemplo, el vídeo de @kylethomas (https://bit.ly/3fRN2CU), teniendo en cuenta que comparte otras categorías de análisis.

5. Vídeos de eventos en los que el tiktoker muestra o narra eventos deportivos, conciertos u otras actividades organizadas a la que han asistido. No se ha registrado ningún vídeo de esta categoría.

6. Vídeo tutoriales en los que los y las adolescentes se muestran guiando un proceso o procedimiento que deben realizar sus seguidores a la hora de realizar una determinada actividad como jugar, maquillarse o recomendaciones sobre cómo incrementar la resistencia física mediante alguna práctica deportiva. Por ejemplo, el vídeo de @ martindant (https://bit.ly/3uin5R8), teniendo en cuenta que comparte otras categorías de análisis. 
7. Vídeos con animales o en los que el animal o la mascota que el adolescente muestra a cámara co-protagoniza el contenido. Por ejemplo, el vídeo de @hanamartinx (https://bit.ly/3unOVLK), teniendo en cuenta que comparte otras categorías de análisis.

8. Videoselfies basados en producciones audiovisuales informales sin guion en la que el influencer se muestra de manera auténtica, con el objetivo de generar impacto positivo en sus seguidores mediante la adquisición del rol protagónico por el modo en él que posa o por cómo se presenta o expresa ante la cámara. Por ejemplo, el vídeo de @abe.oficial (https://bit.ly/3mv1eU0), teniendo en cuenta que comparte otras categorías de análisis.

\section{Resultados}

En el registro de las producciones audiovisuales se advierte que todos los vídeos analizados comparten dos o más tipologías de contenidos llegando a 958 tipos de contenidos, lo que evidencia la dificultad de estudio que entrañan este tipo de investigaciones cuantitativas. Asimismo, se observa que ningún vídeo subido por los adolescentes de ambas nacionalidades pertenece al tipo de contenidos de eventos en el que los protagonistas cuentan o muestran algún evento al que han asistido. Además, los españoles tampoco abordan la categoría de vídeos con animales y los británicos producciones relativas a videojuegos. Esta modificación de las propuestas planteadas por los trabajos científicos explorados pone de manifestó la evolución de los contenidos audiovisuales que están creando y difundiendo los adolescentes en sus canales de las redes sociales así como la continua necesidad de investigación y actualización de la realidad digital en la que están creciendo los adolescentes.

\subsection{Tipología de contenidos de tiktokers españoles}

Los tiktokers españoles han difundido 240 producciones audiovisuales. Los vídeos de los adolescentes españoles son videoselfies (52\%) y vídeos de coreografías y bailes (32\%). Los adolescentes españoles también crean y difunden vídeos cuya finalidad es entretener mediante bromas o acciones divertidas (12\%) y realizando acciones diarias o convencionales (4\%) como haciendo los deberes o preparándose para salir. Por género son los chicos de 17 años los que más contenidos publican con un $25 \%$ de la producción, seguidos de las chicas con un $17 \%$ del total.

Con el objetivo de estudiar si el género y la edad están relacionadas con la creación y difusión de estos contenidos audiovisuales, se analizan por separado la variables de género y edad para estimar si alguna de las dos presenta mayor asociación con el tipo de contenido publicados. En primer lugar, se agrupan las observaciones por género y se observa que chicos y chicas suben similar número de tipo de contenidos en sus producciones (Tabla 2).

Tabla 2. Distribución de observaciones por tipo de contenidos y género

\begin{tabular}{|c|c|c|c|c|}
\hline \multirow[b]{2}{*}{ Tipo de contenidos } & & \multicolumn{2}{|c|}{ Género } & \multirow[b]{2}{*}{ Totales } \\
\hline & & Chica & Chico & \\
\hline Videojuego & & 0 & 1 & 1 \\
\hline Coreografías y vídeos musicales & & 73 & 72 & 145 \\
\hline Vídeos escenas convencionales & & 11 & 7 & 18 \\
\hline Vídeos divertidos & & 24 & 30 & 54 \\
\hline Tutoriales & & 2 & 0 & 2 \\
\hline \multirow[t]{3}{*}{ Videoselfies } & & 118 & 117 & 235 \\
\hline & Totales & 228 & 227 & 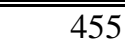 \\
\hline & Porcentaje & $50 \%$ & $50 \%$ & \\
\hline
\end{tabular}




\section{Fuente: elaboración propia}

Aplicando la prueba de chi-cuadrado para analizar si existe relación entre género y el tipo de contenidos, el resultado $\left(\chi^{2}(5)=4,565, p=0,471\right.$ muy inferior al valor crítico de $\chi^{2}=$ 11,070) muestra que ambas variables no guardan una relación de dependencia, con un nivel de confianza del $95 \%$. Estos resultados indican que el género no determina la creación y difusión de los vídeos que se viralizan en esta red social.

En segundo lugar, se investiga si la edad representa una variable significativa en la tipología de vídeos que los adolescentes suben a esta red social y destaca que los adolescentes de 16 y 17 años suben el $49 \%$ de los vídeos seguidos de los adolescentes de 14 años con un 26\% del total (Tabla 3 ).

Tabla 3. Distribución de observaciones por tipo de contenidos y edad

\section{Edad}

\begin{tabular}{|c|c|c|c|c|c|c|}
\hline Tipo de contenidos & 11 & 14 & 15 & 16 & 17 & Totales \\
\hline Videojuego & 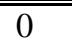 & 0 & 0 & 0 & 1 & 1 \\
\hline Coreografías y vídeos musicales & 11 & 45 & 26 & 25 & 38 & 145 \\
\hline Vídeos escenas convencionales & 1 & 1 & 7 & 6 & 3 & 18 \\
\hline Vídeos divertidos & 6 & 12 & 5 & 10 & 21 & 54 \\
\hline Tutoriales & 0 & 0 & 2 & 0 & 0 & 2 \\
\hline Videoselfies & 21 & 59 & 38 & 40 & 77 & 235 \\
\hline Totales & 39 & 117 & 78 & 81 & 140 & 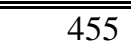 \\
\hline Porcentaje & $9 \%$ & $26 \%$ & $17 \%$ & $18 \%$ & $31 \%$ & \\
\hline
\end{tabular}

Fuente: elaboración propia

Los valores de chi-cuadrado $\left(\chi^{2}(20)=30,717, p=0,06\right)$ confirman que, con un valor crítico de $\chi^{2}=31,410$, la edad no está relacionada con el tipo de contenido publicado. Por lo que la tipología de contenidos en las producciones audiovisuales no está asociada con la edad y el género de los tiktokers españoles.

\subsection{Tipología de contenidos de tiktokers británicos}

Los tiktokers británicos han creado y difundido 237 vídeos. Los vídeos de los adolescentes británicos son videoselfies (50\%), coreografías y videos musicales (25\%) y vídeos divertidos (20\%). Por género, son los chicos de 16 años los que más contenidos publican dentro de las categorías seleccionadas (36\%), seguidos de las chicas de 17 años con un $24 \%$ del total y los chicos de 17 años con un $17 \%$ del total.

Para ofrecer una radiografía de los vídeos que suben a sus cuentas, al igual que en el análisis de los tiktokers españoles, se comprueba si alguna de las variables (género y edad) presenta mayor asociación con el tipo de contenidos publicados. Al igual que sucede para los españoles, chicos y chicas británicos publican vídeos de coreografías, vídeos musicales y videoselfies, pero en este caso son ellos los que más vídeos divertidos suben sus canales de TikTok (chicos 23\% n=67; chicas 17\% $\mathrm{n}=36$ ). En primer lugar, se estudian las observaciones en relación al género y se comprueba que son los chicos británicos (57\%) los que mayor número de contenidos incorporan en sus vídeos (Tabla 4). 
Tabla 4. Distribución de observaciones por tipos de contenidos y género

\begin{tabular}{lccrr} 
& \multicolumn{2}{c}{ Género } & \\
\cline { 2 - 4 } Tipo de contenidos & chica & chico & Totales \\
\hline \hline Coreografias y vídeos musicales & 58 & 72 & 130 \\
Vídeos escenas convencionales & 1 & 5 & 6 \\
Vídeos divertidos & 36 & 67 & 103 \\
Tutoriales & 1 & 5 & 6 \\
Vídeos con animales & 4 & 5 & 9 \\
Videoselfies & 114 & 135 & 249 \\
\hline \hline & Totales & 214 & 289 & 503 \\
& Porcentaje & $43 \%$ & $57 \%$ & \\
\hline
\end{tabular}

Fuente: elaboración propia

Para analizar la relación entre género y tipo de contenidos, la prueba de chi-cuadrado, con un nivel de confianza del $95 \%$, revela $\left(\chi^{2}(5)=7,027, p=0,219\right.$ muy inferior al valor crítico de $\chi^{2}=$ $11,070)$ que las variables de género y el tipo de contenidos publicados no están relacionadas.

En segundo lugar, se agrupan las observaciones por tipo de contenidos y edad y, al igual que los adolescentes españoles, son los centennials de 16 y 17 años los que mayor productividad presentan alcanzando el $71 \%$ del tipo de vídeos (Tabla 5).

Tabla 5. Distribución de observaciones por tipo de contenidos y edad

\begin{tabular}{|c|c|c|c|c|c|c|}
\hline \multirow[b]{2}{*}{ Tipo de contenidos } & \multicolumn{5}{|c|}{ Edad } & \multirow[b]{2}{*}{ Totales } \\
\hline & 12 & 14 & 15 & 16 & 17 & \\
\hline Coreografias y vídeos musicales & 16 & 19 & 16 & 17 & 62 & 130 \\
\hline Vídeos escenas convencionales & 0 & 0 & 0 & 3 & 3 & 6 \\
\hline Vídeos divertidos & 15 & 0 & 6 & 43 & 39 & 103 \\
\hline Tutoriales & 0 & 0 & 0 & 1 & 5 & 6 \\
\hline Vídeos con animales & 0 & 0 & 0 & 5 & 4 & 9 \\
\hline \multirow[t]{3}{*}{ Videoselfies } & 36 & 20 & 20 & 57 & 116 & 249 \\
\hline & 67 & 3 & & 126 & 229 & 503 \\
\hline & $13 \%$ & $8 \%$ & & $25 \%$ & $46 \%$ & \\
\hline
\end{tabular}

Fuente: elaboración propia

Para confirmar la existencia de relación estadística entre la edad y el tipo de vídeos publicados en sus cuentas de TikTok, el resultado de aplicar el valor chi-cuadrado a la tabla anterior es $\chi^{2}(20)=$ $53,607, p<0,01$, que en este caso superior al valor crítico de $\chi^{2}=31,410$, permite concluir la relación de dependencia entre ambas variables, y que es la edad la que determina qué tipo de contenidos deciden crear y difundir los adolescentes británicos.

\subsection{Comparativa tiktokers españoles-británicos: género y edad en los vídeos creados y difundidos}

Para comprobar qué tipología de contenidos están subiendo los adolescentes centennials de ambas nacionalidades se desagregan las variables de género, edad y nacionalidad. En primer lugar, en relación con el género de los autores (Tabla 6) se observa que los chicos de ambas nacionalidades 
(54\%) son los que mayor cantidad de tipo de contenidos integran en sus vídeos. Para confirmar si el género está relacionado estadísticamente con los contenidos que publican, la prueba de chi-cuadrado de Pearson, con un nivel de confianza del 95\%, $\left(\chi^{2}(6)=6,092, p=0,413\right)$ pone de manifiesto la relación de independencia entre las variables analizadas.

Tabla 6. Distribución de observaciones por tipo de contenidos y edad

\begin{tabular}{|c|c|c|c|c|}
\hline \multirow[b]{2}{*}{ Tipos de contenidos } & \multicolumn{3}{|c|}{ Género } & \multirow[b]{2}{*}{ Totales } \\
\hline & chica & & chico & \\
\hline Videojuego & 0 & & 1 & 1 \\
\hline Coreografías y videos musicales & 131 & & 144 & 275 \\
\hline Vídeos escenas convencionales & 12 & & 12 & 24 \\
\hline Vídeos divertidos & 60 & & 97 & 157 \\
\hline Tutoriales & 3 & & 5 & 8 \\
\hline Vídeos con animales & 4 & & 5 & 9 \\
\hline \multirow{3}{*}{$\begin{array}{r}\text { Totales } \\
\text { Porcentaje }\end{array}$} & 232 & & 252 & 484 \\
\hline & & 442 & 516 & 958 \\
\hline & & $46 \%$ & $54 \%$ & \\
\hline
\end{tabular}

Fuente: elaboración propia

En el siguiente estadio del análisis se agrupan las edades y el tipo de contenidos. En consonancia con los resultados del análisis individual por nacionalidad, se confirma que el $61 \%$ de la producción total de ambas nacionalidades los crean y difunden los adolescentes de 16 y 17 años centrándose videoselfies $(50 \% \mathrm{n}=290)$, vídeos con coreografías y vídeos musicales $(35 \% \mathrm{n}=142)$ y vídeos divertidos (20xx\% $\mathrm{n}=113)$ (Tabla 7). Para conocer si la edad resulta determinante en las producciones audiovisuales que los centennials incorporan en sus vídeos, se estudia la frecuencia de relación entre ambas variables mediante el cálculo del valor chi-cuadrado cuyo resultado $\left(\chi^{2}(30)=\right.$ $68,094, p>0,001$, superior al valor crítico $\chi^{2}=43,773$.) muestra la asociación de dependencia entre las dos variables lo que confirma que dependiendo de su edad se decantan por publicar un tipo de contenido u otro.

Tabla 7. Distribución de observaciones por tipo de contenidos y edad

\begin{tabular}{|c|c|c|c|c|c|c|c|}
\hline \multirow[b]{2}{*}{ Tipos de contenidos } & \multicolumn{6}{|c|}{ Edad } & \multirow[b]{2}{*}{ Totales } \\
\hline & 11 & 12 & 14 & 15 & 16 & 17 & \\
\hline Videojuego & 0 & 0 & 0 & 0 & 0 & 1 & 1 \\
\hline Coreografías y videos musicales & 11 & 16 & 64 & 42 & 42 & 100 & 275 \\
\hline Vídeos escenas convencionales & 1 & 0 & 1 & 7 & 9 & 6 & 24 \\
\hline Vídeos divertidos & 6 & 15 & 12 & 11 & 53 & 60 & 157 \\
\hline Tutoriales & 0 & 0 & 0 & 2 & 1 & 5 & 8 \\
\hline Vídeos con animales & 0 & 0 & 0 & 0 & 5 & 4 & 9 \\
\hline Videoselfies & 21 & 36 & 79 & 58 & 97 & 193 & 484 \\
\hline Totales & 39 & 67 & 156 & 120 & 207 & 369 & 958 \\
\hline Porcentaje & $4 \%$ & $7 \%$ & $16 \%$ & $13 \%$ & $22 \%$ & $39 \%$ & \\
\hline
\end{tabular}

Fuente: elaboración propia

Por último, exploramos la existencia de relación entre los contenidos publicados por los adolescentes y su nacionalidad. Los españoles se decantan por crear vídeos de coreografías y vídeos musicales (53\% del total), seguidos de videoselfies (49\% del total) y vídeos divertidos (34\% del total). En el 
caso de los británicos lideran los resultados los vídeos divertidos (66\% del total), videoselfies (51\% del total) y vídeos musicales con coreografías (47\% del total). Resulta destacable señalar que ningún adolescente español comparte vídeos con mascotas frente a 9 de los británicos, y que solo un vídeo pertenece a la categoría de videojuegos en el que un tiktoker español aparece jugando, lo cual puede indicar que esta red social no es la preferida para compartir este tipo de contenido (Tabla 8).

Tabla 8. Distribución de observaciones por tipo de contenidos y nacionalidad

\begin{tabular}{|c|c|c|c|c|c|}
\hline \multirow[b]{3}{*}{ Tipos de contenidos } & \multicolumn{5}{|c|}{ Nacionalidad } \\
\hline & \multirow[b]{2}{*}{ Española } & \multirow[b]{2}{*}{ Inglesa } & \multirow[b]{2}{*}{ Totales } & \multicolumn{2}{|l|}{$\%$} \\
\hline & & & & Española & $\%$ Inglesa \\
\hline Videojuego & 1 & 0 & 1 & $100 \%$ & $0 \%$ \\
\hline Coreografías y vídeos musicales & 145 & 130 & 275 & $53 \%$ & $47 \%$ \\
\hline Vídeos escenas convencionales & 18 & 6 & 24 & $75 \%$ & $25 \%$ \\
\hline Vídeos divertidos & 54 & 103 & 157 & $34 \%$ & $66 \%$ \\
\hline Tutoriales & 2 & 6 & 8 & $25 \%$ & $75 \%$ \\
\hline Videos con animales & 0 & 9 & 9 & $0 \%$ & $100 \%$ \\
\hline Videoselfies & 235 & 249 & 484 & $49 \%$ & $51 \%$ \\
\hline Totales & 455 & 503 & 958 & & \\
\hline Porcentaje & $47 \%$ & $53 \%$ & & & \\
\hline
\end{tabular}

Fuente: elaboración propia

Para acreditar la validez de las conclusiones estadísticas y conocer si existe relación significativa entre la nacionalidad y el tipo de contenidos, se aplica la prueba de chi-cuadrado de Pearson $(\chi \mid 2)$ con un nivel de confianza del 95\%. El resultado obtenido $\left(\chi^{2}(6)=32,19, p<0,001\right)$ es superior al valor teórico 12,592 lo que corrobora la relación estadística entre el tipo de contenidos y la nacionalidad de los autores de los vídeos. Dado que existe relación entre ambas variables, se realiza la prueba ANOVA de un factor ampliada mediante el test Brown-Forsythe con el objetivo de conocer si el número de contenidos por tipo difiere según la nacionalidad de los adolescentes. El resultado indica $(\mathrm{F}(1,12)=0,019, p=0,892$, ) que no se registran evidencias de relación de dependencia entre las variables, que confirma la equiparación en la creación y difusión de los tiktokers de ambas nacionalidades.

\section{Conclusiones y discusión}

El estudio de esta red social se revela paradigmático para comprender los contenidos que están subiendo, difundiendo y visionando los adolescentes en esta nueva red social. Esta investigación revela que la producción y difusión de los centennials españoles y británicos en TikTok se centra principalmente en videoselfies, coreografías y contenidos divertidos cuya finalidad es entretener a sus seguidores solos o acompañados de amigos o familiares. Esta homogeneización de contenidos confirma que la creación y visualización de contenidos audiovisuales en TikTok no garantiza la pluralidad de contenidos a los que pueden acceder los adolescentes, sino que pone de manifiesto la uniformidad de las producciones de los adolescentes, en línea con los resultados de Masip, et al., (2015) que señalan una estandarización de contenidos en la información de actualidad y las redes sociales , y con Zhu, et al., (2020) en los vídeos publicados en TikTok de contenidos de salud.

Los resultados acreditan que del total de tipo de contenidos en TikTok, el $71 \%$ no son solo coreografías y vídeos musicales basados en la interpretación de fragmentos musicales, lo que pone de manifiesto que los adolescentes están añadiendo a sus vídeos funcionalidades de autoexpresión y 
representación según sus intereses y afinidades (Angulo, 2019). Estas prácticas suceden en línea con la teoría de Goffman (2009) de la presentación de la persona en la vida cotidiana aplicada a las interacciones en las redes sociales en el contexto de una nueva sociabilidad virtual (Torres, 2016). Los adolescentes definen el rol que van a simbolizar, el escenario, la acción y la representación mostrándose en un escenario de actuaciones sociales y escenificación digital (Yarosh, et al., 2016) con el objetivo de crear impresiones en su público y de reflejar una identidad pretendidamente favorable que, como señalan McRoberts, et al., (2017), puede derivar en la creación de contenidos superficiales.

Por géneros se identifica que los chicos y las chicas de 16 y 17 años de ambas nacionalidades son los más activos en esta red social. Siendo los chicos británicos los que mayor cifra de tipo de vídeos incorporan en sus producciones (españoles, 49\%; británicos 71\%). Destaca que los chicos españoles y británicos renuncian a los videojuegos modificando su auto-representación digital en esta red social desenterrando su funcionalidad hegemónica como gameplayers, como no ocurre en otras redes sociales (Kaur y Kaur, 2017; Lange y Schwab, 2018; Maloney, Roberts y Caruso, 2018). El motivo quizá sea la corta duración de los videos, que con una duración máxima de 60 segundos, no les posibilita explayase en las narrativas de las partidas que requieren mayor extensión. Esta evolución de los contenidos que comparten los varones sugiere la transmutación de los gustos e intereses que manifiestan en esta red social así como la influencia que la misma plataforma ejerce sobre ellos en relación a las propias limitaciones técnicas de la plataforma y a la homogenización de contenidos comentada.

A la luz del estudio estadístico realizado podemos concluir que la edad, y no el género, y la nacionalidad muestran mayor relación estadística con los tipos de contenidos publicados y determinan el tipo de contenidos que publican en el caso de los adolescentes británicos y en la comparativa realizaba de ambas nacionalidades. Estos resultados apuntan a una evolución de los roles tradicionales de género que se han estudiado a otras redes sociales como YouTube e Instagram (Linares-Bahillo, Aristegui-Fradua y Beloki-Marañón, 2019; Regueira, Alonso-Ferreiro y Da-Vila, 2020; Ye, et al., 2018). Nuestros resultados sugieren que los y las adolescentes en esta red social muestran comportamientos similares en relación a los contenidos que producen y difunden dando lugar al desvanecimiento de sus diferencias de género, en consonancia con las investigaciones de van Oosten y Vandenbosch (2017) que confirman la igualdad de género en las representación estereotipadas de adolescentes en las redes sociales y de Korlat, et al (2021) que descubren más similitudes que diferencias de género en las autodescripciones que los adolescentes realizan de sí mismos en la redes sociales.

\section{Futuras investigaciones}

Para próximas investigaciones resultará interesante incrementar la muestra para corroborar los resultados obtenidos. Asimismo, será necesario estudiar las motivaciones de los adolescentes para decantarse por una red social u otra así como la finalidad que le otorgan a cada una. Además, ante la facilidad de acceso a las cuentas de menores en TikTok, se pone de manifiesto la trascendencia de examinar los riesgos y peligros a los que están expuestos los menores, sus vulnerabilidades así como la mediación parental y la influencia de los pares en una red social desarrollada exclusivamente para dispositivos móviles. También resultará relevante formalizar investigaciones adicionales sobre las políticas publicitaras de la red social con las que los tiktokers pueden ingresar beneficios de sus creaciones audiovisuales. 


\section{Referencias}

Aguaded, J. I., \& Sánchez Carrero, J. (2013). El empoderamiento digital de niños y jóvenes a través de la producción audiovisual. AdComunica, 5. 175-196. https://doi.org/10.6035/109

Ahlse, J., Nilsson, F., \& Sandström, N. (2020). It's time to TikTok: Exploring Generation Z's motivations to participate in \#Challenges. Bachelor Thesis. Jönköping University. https://www.diva-portal.org/smash/get/diva2:1434091/FULLTEXT01.pdf.

Anderson, K. E. (2020). Getting acquainted with social networks and apps: it is time to talk about TikTok. Library Hi Tech News.

Anggreni, L. S., Redfada, G. D., Yuliarti, M. S., \& Anis, M. Y. (2019). Teenagers and Instagram: The Impact of Becoming Instagram High Users among High School Students in Surakarta. JURNAL ILMU KOMUNIKASI, 2(2), 23-33.

Angulo, M. (2019). Cambios y continuidades en la transformación digital de los medios de comunicación peruanos. Análisis de las tesis peruanas sobre periodismo digital. Emprende y Transforma, 1 (1), 11-20. https://doi.org/10.33829/emprendeytrans- forma-0101-2019-11-20

Baños-González, M., Canorea Tiralaso, H. \& Rajas Fernández, M. (2020). La difusión del vídeo musical en YouTube. Análisis de la capacidad viral del vídeoclip. Revista Latina de Comunicación Social, (77), 117-141. https://www.doi.org/10.4185/RLCS-2020-1452

Blanco-Ruiz, M., \& Sainz-de-Baranda, C. (2018). Channels produced by LGBT+ Youtubers: gender discourse analysis. Observatorio (OBS*), 12(SPE1), 97-121. https://bit.ly/2GnNUzz

Bleakley, A., Ellithorpe, M., \& Romer, D. (2016). The role of parents in problematic internet use among US Adolescents. Media and Communication, 4 (3), 24-34. http://dx.doi.org/10.17645/mac.v4i3.523

Booker, C. L., Kelly, Y. J., \& Sacker, A. (2018). Gender differences in the associations between age trends of social media interaction and well-being among 10-15 year olds in the UK. BMC Public Health, 18(1), 1-12. https://doi.org/10.1186/s12889-018-5220-4

Bossen, C. B., \& Kottasz, R. (2020). Uses and gratifications sought by pre-adolescent and adolescent TikTok consumers. Young Consumers. 21(4), 1747-3616. https://doi.org/10.1108/YC-07-2020$\underline{1186}$

Botou, A., \& Marsellos, P. S. (2018). Teens' Perception about Social Networking Sites: Does Facebook Influence Teens' Self-Esteem? Psychology, 9(06), 1453. https://doi.org $\underline{10.4236 / \text { psych.2018.96089 }}$

Bresnick, E. (2019). Intensified Play: Cinematic study of TikTok mobile app. www.researchgate.net/publication/335570557_Intensified_Play_Cinematic_study_of_TikTok_mo $\underline{\text { bile_app }}$

Bruna, Y. (2020). Snapchat in teenage years. Reseaux, (4), 139-164. https://doi.org/10.3917/res.222.0139 
Catalina-García, B., Sousa, J. P., \& Sousa, L. C. (2019). Consumo de noticias y percepción de fake news entre estudiantes de Comunicación de Brasil, España y Portugal. Observatorio (OBS*) Revista de comunicación, 18(2), 52-69. http://dx.doi.org/1646-5954/ERC123483/2019

Costa, C. (2013). Narrativas transmedia nativas: ventajas, elementos de la planificación de un proyecto audiovisual transmedia y estudio de caso. Historia y Comunicación Social, 18, 561-574. http://dx.doi.org/10.5209/rev_HICS.2013.v18.44349

Djafarova, E. \& Rushworth, C. (2017). Exploring the credibility of online celebrities' Instagram profiles in influencing the purchase decisions of young female users. Computers in Human Behaviour, 68, 1-7. https://doi.org/10.1016/j.chb.2016.11.009

Djafarova, E., \& Trofimenko, O. (2019). 'Instafamous'-credibility and self-presentation of microcelebrities on social media. Information, communication y society, 22(10), 1432-1446. https://doi.org/10.1080/1369118X.2018.1438491

Edelmann, N. (2017). Lurking in online participation and e-participation. In 2017 Fourth International Conference on eDemocracy y eGovernment (ICEDEG). 282-284. IEEE. http://doi.org/10.1109/ICEDEG.2017.7962552

Fernández de Arroyabe, M. A., Lazkano-Arrillaga, I., \& Eguskiza-Sesumaga, L. (2018). Nativos digitales: Consumo, creación y difusión de contenidos audiovisuales online. Comunicar, 57, 6169. https://doi.org/10.3916/C57-2018-06

Fondo de las Naciones Unidas para la Infancia (UNICEF, 2020). Adolescencia y juventud. https://www.unicef.org/spanish/adolescence/index_bigpicture.html

García Jiménez, A., y Montes Vozmediano, M. (2020). Subject matter of videos for teens on YouTube. International Journal of Adolescence and Youth, 25(1), 63-78. https://doi.org/10.1080/02673843.2019.1590850

García-Jiménez, A., López-de-Ayala López, M., y Montes-Vozmediano, M. (2020). Características y percepciones sobre el uso de las plataformas de redes sociales y dispositivos tecnológicos por parte de los adolescentes. Zer, 25(48), 269-286. https://doi.org/10.1387/zer.21556.

Gazit, T., Bronstein, J., Amichai-Hamburger, Y., Aharony, N., Bar-Ilan, J. \& Pérez, O. (2018). Active participants and lurkers in online discussion groups: an exploratory analysis of focus group interviews and observation. Information Research, 23(2), paper 791. http://InformationR.net/ir/23-2/paper791.html

Goffman, E. (2009). La presentación de la persona en la vida cotidiana. Buenos Aires: Amorrortu.

Gómez, O. (2018). Análisis de la narrativa audiovisual de los Youtubers y su impacto en los jóvenes colombianos (Doctoral dissertation, Tesis de doctorado). Universidad de Málaga, Málaga, España.

Gonzales, A. L., \& Hancock, J. T. (2011). Mirror, mirror on my Facebook wall: Effects of exposure to Facebook on self-esteem. Cyberpsychology, behavior, and social networking, 14(1-2), 79-83. https://doi.org/10.1089/cyber.2009.0411 
Haddon, L., \& Vincent, J. (2015). UK children's experience of smartphones and tablets: Perspectives from children, parents and teachers. London: LSE. http://netchildrengomobile.eu/reports/

Holtzman, S. (1997). Digital Mosaics: The Aesthetics of Cyberspace. New York: Touchstone.

Hubermann, S. (2020). Images on Social Media, Their Producers and Viewers. KULT_online, (62).

Jauregui Caballero, A. \& Ortega Ponce, C. (2020). Narrativas transmediáticas en la apropiación social del conocimiento. Revista Latina de Comunicación Social, (77), 357-372. https://www.doi.org/10.4185/RLCS-2020-1462

Kaur, L., \& Kaur, J. (2017). Gender difference in video game habits among adolescents. International Journal of Education and Management Studies, 7(4), 512-514. http://www.iahrw.com/index.php/home/journal_detail/21\#list

Khattab, M., (2019). Synching and performing: body (re)- presentation in the short video app TikTok. WiderScreen 21(1-2). https://bit.ly/36hiPYA

Klug, D. (2020). "It took me almost 30 minutes to practice this". Performance and Production Practices in Dance Challenge Videos on TikTok. https://arxiv.org/ftp/arxiv/papers/2008/2008.13040.pdf

Korlat, S., Forst, N., Schultes, M., Schober, M. T., Spiel, B., \& Kollmayer, M. (2021). Gender role identity and gender intensification: Agency and communion in adolescents' spontaneous selfdescriptions. European Journal of Developmental Psychology, 1-25. https://doi.org/10.1080/17405629.2020.1865143

Krippendorff, K. (2018). Content analysis: An introduction to its methodology. Sage publications.

Lange, B. P., \& Schwab, F. (2018). Game on: Sex differences in the production and consumption of video games. Evolutionary psychology and digital games: Digital hunter-gatherers. New York, NY: Routledge.

Leight, E. (2019). If you can get famous easily, you're gonna do it": how TikTok took over music. Rolling Stone. https://www.rollingstone.com/pro/features/tiktok-video-app-growth-867587/

Lenhart, A. (2015). Teens, social media, y technology overview 2015. Pew Research Center, Internet and Technology. https://www.pewresearch.org/internet/2015/04/09/teens-social-mediatechnology-2015/

Linares-Bahillo, E.; Aristegui-Fradua, I. \& Beloki-Marañón, U. (2019). YouTube, una plataforma para la (in)formación, relación, comunicación, diversión, y gestión de identidades (de género) en la natividad digital. Revista Mediterránea de Comunicación/Mediterranean Journal of Communication, 10(1), 55-70. https://www.doi.org/10.14198/MEDCOM2019.10.1.18

López de Ayala López, M. C., \& Paniagua Santamaría, P. (2019). Motivaciones de los jóvenes para la creación y difusión de contenido en sitios de redes sociales. Estudios Sobre El Mensaje Periodístico, 25(2), 915-933. https://doi.org/10.5209/esmp.64816 
López, J. (2020). ¿Qué pasó con la red social Vine? Aquí te lo contamos todo. Digitaltrends. https://es.digitaltrends.com/sociales/que-paso-con-la-red-social-vine/

Luque, S. G., Tejedor, S., \& Bugs, R. C. (2017). Motivaciones de uso de las redes sociales de los estudiantes de periodismo de América Latina y España. Informação y Sociedade, 27(3). 191-203. https://brapci.inf.br/_repositorio/2018/01/pdf_51422c9d21_0000029217.pdf

Maloney, M., Roberts, S., \& Caruso, A. (2018). 'Mmm... I love it, bro!': Performances of masculinity in YouTube gaming. New Media y Society, 20(5), 1697-1714. https://doi.org/10.1177/1461444817703368

Marcelino, G. V. (2015). Migración de los jóvenes españoles en redes sociales, de Tuenti a Facebook $\mathrm{y}$ de Facebook a Instagram. La segunda migración. Icono14, 13(2), 48-72. http://doi.org/10.7195/ri14.v13i2.821

Martín. A. (2020). Facebook echa el cierre a Lasso, su copia de TikTok. Hipertextual. https://hipertextual.com/2020/07/facebook-lasso-cierre

Masip, P., Guallar, J., Suau, J., Ruiz-Caballero, C., \& Peralta, M. (2015). News and social networks: audience behavior. El profesional de la información, 24, (4), 363-370. http://dx.doi.org/10.3145/epi.2015.jul.02

McGorry, S. Y., \& McGorry, M. R. (2017). Who are the Centennials: Marketing Implications of Social Media Use and Preferences. https://bit.ly/3dZDlkW

McMillan, W. J. (2009). Finding a method to analyze qualitative data: using a study of conceptual learning. Journal of dental education, 73(1), 53-64. https://doi.org/10.1002/j.00220337.2009.73.1.tb04639.x

McRoberts, S., Ma, H., Hall, A., \& Yarosh, S. (2017). Share first, save later: Performance of self through Snapchat stories. In Proceedings of the 2017 CHI conference on human factors in computing systems, 6902-691. http://dx.doi.org/10.1145/3025453.3025771

Montes-Vozmediano, M., García-Jiménez, A., \& Menor-Sendra, J. (2018). Los vídeos de los adolescentes en YouTube: Características y vulnerabilidades digitales. Comunicar, 26(54), 61-69. https://doi.org/10.3916/C54-2018-06

Patel, K. \& Binjola, H. (2020). Tik Tok los nuevos medios alternativos para jóvenes para compartir talento en línea: un estudio analítico. SSRN. http://dx.doi.org/10.2139/ssrn.3600119

Pedraza Moreno, K. \& Villarraga Castañeda, S. E. (2019). Los YouTubers: espejos influyentes en el proyecto de vida adolescente. Imaginario Social, 2(2), 12-27. http://www.revistaimaginariosocial.com/index.php/es/article/view/13/23

Pérez-Rufí, J. P. (2018). Producción y edición del vídeo de bajo presupuesto: estudio de caso de "Lost Ones" (Joan Thiele) de Giada Bossi. Revista Mediterránea de Comunicación/Mediterranean Journal of Communication, 9(2), 291-303. https://www.doi.org/10.14198/MEDCOM2018.9.2.4 
Qasem, Z. (2021). The effect of positive TRI traits on centennials adoption of try-on technology in the context of E-fashion retailing. International Journal of Information Management, 56. https://doi.org/10.1016/j.ijinfomgt.2020.102254

Qustodio (2020). Apps y nativos digitales: la nueva normalidad. https://www.observatoriodelainfancia.es/oia/esp/documentos_ficha.aspx?id=7131

Regueira, U., Alonso-Ferreiro, A., \& Da-Vila, S. (2020). La mujer en YouTube: Representación y participación a través de la técnica Web Scraping. Comunicar: Revista Científica de Comunicación y Educación, 28(63), 31-40. https://doi.org/10.3916/C63-2020-03

Richards, L. (2020). Handling qualitative data: A practical guide. Sage Publications Limited.

Ródenas, Ferreras \& Torrado (2021). Introducción: Bienvenidos a la cultura de la convergencia. En: Torrado, Roderas y Ferreras (eds). Territorios transmedia y narrativas audiovisuales, 15-17. Barcelona: Editorial UOC.

Romero-Hall, E. (2017). Active user or lurker? A phenomenological investigation of graduate students in social media spaces. International Journal of Social Media and Interactive Learning Environments, 5(4), 326-340. http://dx.doi.org/10.5027/10.1504/IJSMILE.2017.090975

Sensortower, (2020). Top Apps Worldwide for May 2020 by Downloads. https://sensortower.com/blog/top-apps-worldwide-may-2020-downloads

Sharma, A. (2019). Consumer behaviour and centennials. In International Conference on Marketing. Marketing to Centennials in Digital World. 37-49.

Sherman, L. E, Payton, A., Hernandez, L. M., Greenfield, P.M. \& Dapretto, M. (2016). The power of the like in adolescence: Effects of peer influence on neural and behavioral responses to social media. Psychological Science, 27 (7), 1027-1035, http://dx.doi.org/10.1177/0956797616645673

Sherman, L. E., Greenfield, P.M., Hernandez, L.M \& Dapretto, M. (2018). Peer influence via instagram: Effects on brain and behavior in adolescence and young adulthood. Child Development, 89 (1), 37-47. http://dx.doi.org/10.1111/cdev.12838|

Shutsko A. (2020) User-Generated Short Video Content in Social Media. A Case Study of TikTok. In: Meiselwitz G. (eds) Social Computing and Social Media. Participation, User Experience, Consumer Experience, and Applications of Social Computing. HCII 2020. Lecture Notes in Computer Science, vol 12195. Springer, Cham. https://doi.org/10.1007/978-3-030-49576-3_8

Smith, D. P., \& Mills, S. (2019). The 'youth-fullness' of youth geographies:'coming of age'? Children's Geographies, 17, 1-8. https://doi.org/10.1080/14733285.2018.1539701

Statista (2020a). Ranking de aplicaciones de redes sociales y mensajería para Android más populares en Google Play Store en España en enero de 2020, por número de descargas. https://es.statista.com/estadisticas/883635/apps-de-redes-sociales-y-mensajeria-para-android-masdescargadas-espana/ 
Statista (2020b). Average time spent by children daily on TikTok, Instagram, Snapchat and YouTube in the United Kingdom (UK), as of February 2020. https://www.statista.com/statistics/1124962/time-spent-by-children-on-social-media-uk/

Suing, A., Carpio Jiménez, L., \& Sinche, A. (2020). Estética y narrativa audiovisual de youtubers católicos. Questión, 2 (66). https//doi.org/10.24215/16696581e464

Sulistyo, P. B. (2018). Teenagers Preferences, Creations and Participation in Vlogging Activities. International Journal of Scientific and Research Publications (IJSRP), 8(8). http://dx.doi.org/10.29322/IJSRP.8.8.2018.p80113

The Office of Communications (Ofcom, 2020). UK's internet use surges to record levels. https://www.ofcom.org.uk/about-ofcom/latest/features-and-news/uk-internet-use-surges

Torres, C. (2016). Narrativas Mediáticas: La representación virtual del yo en los jóvenes. Realidad: Revista de Ciencias Sociales y Humanidades, 147. 153-168.

Tur-Viñes, V., Núñez-Gómez, P., \& González-Río, M. J. (2021). Menores influyentes en YouTube. Un espacio para la responsabilidad. Revista Latina de Comunicación Social, (73), 1211-1230. https://doi.org/10.4185/RLCS-2018-1303

van Dijck, J. (2019). La cultura de la conectividad: una historia crítica de las redes sociales. Siglo XXI Editores.

van Oosten, J. M., \& Vandenbosch, L. (2017). Sexy online self-presentation on social network sites and the willingness to engage in sexting: A comparison of gender and age. Journal of Adolescence, 54, 42-50. https://doi.org/10.1016/j.adolescence.2016.11.006

Wang, S. (2020b). Personal Branding Strategies of Female Entertainment Influencers on TikTok. Thesis. Rochester Institute of Technology. https://scholarworks.rit.edu/theses/10624

Wang, Y. (2020a). Influence of camera view on TikTok users' presence, immersion, and adoption intent. Computers in Human Behavior, 106373. https://doi.org/10.1016/j.chb.2020.106373

We Are Social y Hootsuite. (2019). Digital 2019. Global Digital Overview. https://bit.ly/322RhmG

We Are Social y Hootsuite. (2020). Digital 2020: Global Digital Overview. https://bit.ly/3gc9kge

We are social. (2020). Digital 2020: The United Kingdom. https://wearesocial.com/uk/digital-2020$\underline{\mathrm{uk}}$

Witt, E. A., Massman, A. J., y Jackson, L. A. (2011). Trends in youth's videogame playing, overall computer use, and communication technology use: The impact of self-esteem and the Big Five

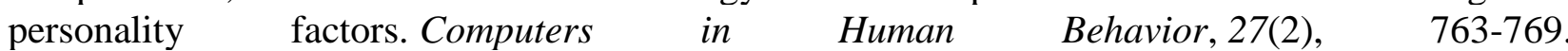
https://doi.org/10.1016/j.chb.2010.10.025

Wotanis, L. \& McMillan, L. (2014). Performing Gender on YouTube: How Jenna Marbles Negotiates a Hostile Online Environment. Feminist Media Studies, 14 (6), 912-928. https://doi.org/10.1080/14680777.2014.882373 
Yang, S., Zhao, Y., \& Ma, Y. (2019). Analysis of the Reasons and Development of Short Video Application-Taking Tik Tok as an Example. In Proceedings of the 2019 9th International Conference on Information and Social Science (ICISS 2019), 12-14. https://doi.org/10.25236/iciss.2019.062

Yarosh, S., Bonsignore, E., McRoberts, S., \& Peyton, T. (2016). YouthTube: Youth video authorship on YouTube and Vine. Proceedings of the 19th ACM Conference on Computer-Supported Cooperative Work y Social Computing, 1423-1437. https://doi.org/10.1145/2818048.2819961

Ye, Z., Hashim, N. H., Baghirov, F., \& Murphy, J. (2018). Gender differences in Instagram hashtag use. Journal of Hospitality Marketing y Management, 27(4), 386-404. https://doi.org/10.1080/19368623.2018.1382415

Zhu, C., Xu, X., Zhang, W., Chen, J., \& Evans, R. (2020). How health communication via TikTok makes a difference: a content analysis of Tik Tok accounts run by Chinese Provincial Health Committees. International journal of environmental research and public health, 17(1), 192. https://doi.org/10.3390/ijerph17010192

Zlamal, R., Machackova, H., Smahel, D., Abramczuk, K., Ólafsson, K., y Staksrud, E. (2020). EU Kids Online 2020: Technical report. EU Kids Online. https://doi.org/10.21953/lse.04dr94matpy7

\section{AUTOR/ES:}

\section{Rebeca Suárez-Álvarez}

Doctora en Comunicación Social y profesora del Departamento de Comunicación y Sociología de la Universidad Rey Juan Carlos. Licenciada en Periodismo por la Universidad Complutense de Madrid (UCM). Posee dos master: uno en radio en la (CEU) y otro de Comunicación de Instituciones Públicas y Políticas (UCM). Miembro del grupo de investigación sobre Comunicación Sociedad y Cultura (GICOMSOC). Sus principales líneas de investigación son la comunicación y los públicos vulnerables (menores), nuevos medios, alfabetización mediática y competencia digital. Ha participado en diferentes proyectos de investigación y en la actualidad, es investigadora en el proyecto "Nuevos escenarios de vulnerabilidad digital: alfabetización mediática para una sociedad inclusiva" (PROVULDIG-2-CM) (ref. H2019/HUM5775), financiado por CAM y Fondo Social Europeo.

rebeca.suarez@urjc.es

Orcid ID: https://orcid.org/0000-0002-0102-4472

Google Scholar: https://scholar.google.es/citations?user=AnYEWykAAAAJyhl=es

ResearchGate: https://www.researchgate.net/profile/Rebeca_Suarez4

Academia.edu: $\underline{\text { https://urjc.academia.edu/RebecaSuarez }}$

\section{Antonio García-Jiménez}

Catedrático de Periodismo en la Universidad Rey Juan Carlos (Departamento de Comunicación y Sociología). Doctor en Ciencias de la Información. Ex Decano de la Facultad de Ciencias de la Comunicación de la Universidad Rey Juan Carlos (2008-2014). Ha sido también director del Máster en Comunicación y Problemas Socioculturales (2015-2018). Profesor en el grado de Periodismo y en varios másteres de Comunicación (Medios Sociales; Periodismo de Datos). Investigador principal del grupo de investigación sobre comunicación, sociedad y cultura (GICOMSOC). Ha dirigido o participado en 19 proyectos de investigación relacionados con los usos y riesgos digitales para los 
RLCS, Revista Latina de Comunicación Social, 79, 1-22

[Investigación] DOI: 10.4185/RLCS-2021-1503| ISSN 1138-5820| Año 2021

adolescentes y es autor de más de 24 contribuciones en forma de libro o capítulos de libro. Es miembro de ECREA, IAMCR y AE+IC.

antonio.garcia@urjc.es

\section{Índice H: 20}

Orcid ID: https://orcid.org/0000-0002-8423-9486

Google Scholar: $\underline{\text { https://scholar.google.es/citations?user=PQomUtAAAAAJyhl=es }}$

ResearchGate: https://www.researchgate.net/profile/Antonio_Garcia_Jimenez2 